\title{
SOLUTION OF PARAXIAL WAVE EQUATION FOR INHOMOGENEOUS MEDIA IN LINEAR AND QUADRATIC APPROXIMATION
}

\author{
ALEX MAHALOV AND SERGEI K. SUSLOV
}

(Communicated by Ken Ono)

\begin{abstract}
We construct explicit solutions of the inhomogeneous parabolic wave equation in a linear and quadratic approximation. As examples, oscillating laser beams in a $1 D$ parabolic waveguide, spiral light beams in $2 D$ varying media and an effect of superfocusing of particle beams in a thin monocrystal film are briefly discussed. Transformations of nonlinear equations into the corresponding autonomous and homogeneous forms are found and a review of important applications is also given.
\end{abstract}

\section{INTRODUCTION}

The inverse scattering method is a standard approach to several completely integrable nonlinear partial differential equations, such as the Korteweg-de Vries, nonlinear Schrödinger, Sine-Gordon and Kadomtsev-Petviashvili equations [1, 2], [60], 81. At the same time, the physical situations in which these equations arise are usually highly idealized. The inclusion of damping, external forces, an inhomogeneous medium with variable density and a higher order of the nonlinearity may provide a more realistic model. Yet, the addition of these perturbation effects could mean that the system is no longer completely integrable (see an example in Refs. 1], 14], 35] and [70]). Hence it is of interest to determine under what conditions the perturbation preserves the integrability. Some of the nonintegrable systems in question possess important classes of exact solutions (see, for instance, [18], 20, 21], 19], 58, 55, 64] and the references therein). These solutions may serve as a starting point of perturbation methods and provide a useful testing ground for numerical investigation of more complicated models including stochastic differential equations [8, [28, 62, 62].

In this paper, we show that the following nonlinear PDE in the two-dimensional space-time continuum:

$$
\frac{\partial \psi}{\partial t}+Q\left(\frac{\partial}{\partial x}, x\right) \psi=P\left(\psi, \psi^{*}, \frac{\partial \psi}{\partial x}, \frac{\partial \psi^{*}}{\partial x}\right),
$$

where $Q$ is a quadratic of two (noncommuting) operators $\partial / \partial x$ and $x$ with timedependent coefficients, the asterisk denotes the complex conjugation, and $P$ is a

Received by the editors March 26, 2013.

2010 Mathematics Subject Classification. Primary 35Q55, 35Q51; Secondary 35C05, 81Q05.

Key words and phrases. Paraxial wave equations, Green's function, Airy-Gaussian-Hermite beams, Gaussian-Hermite beams, nonlinear Schrödinger equations, complex Ginsburg-Landau equations.

This research was partially supported by AFOSR grant FA9550-11-1-0220. 
gauge invariant nonlinearity, can be reduced by a certain change of variables to the autonomous form:

$$
\frac{\partial \chi}{\partial \tau}+\frac{\partial^{2} \chi}{\partial \xi^{2}}=R\left(\chi, \chi^{*}, \frac{\partial \chi}{\partial \xi},\left(\frac{\partial \chi}{\partial \xi}\right)^{*}\right)
$$

which is somewhat easier to analyze by a variety of available tools. In general, the new time variable $\tau$ may be complex-valued and the new "spacial" variable $\xi$ is a linear complex-valued function of $x$ (the precise form of this transformation is established by Theorem 1). Our approach can also be used for nonlinear equations with some stochastic coefficients and random initial data. Among important applications are the nonlinear parabolic equation of diffraction theory, the derivative nonlinear Schrödinger equation, which describes the propagation of circular polarized nonlinear Alfvén waves in plasma physics, the quintic complex GinzburgLandau equation, and the Gerdjikov-Ivanov equation. (We mainly concentrate on variants of the nonlinear Schrödinger equation for which the unperturbed models are known to be completely integrable and/or have explicit solutions.)

The paper is organized as follows. In sections 2 to 4 , we study the inhomogeneous linear parabolic equation of the theory of electromagnetic wave diffraction in varying media. In quadratic approximation with respect to the spacial variables perpendicular to the direction of the wave propagation and their gradient, we solve this equation in terms of the corresponding Fresnel integral. Explicit solutions are constructed from the given fields on the boundary by using the Green function of generalized harmonic oscillators extensively studied in quantum mechanics. Certain oscillating laser beams in a $1 D$ parabolic waveguide and spiral beams in $2 D$ varying media are briefly discussed as examples. In section 5 , we transform equation (1.1) into the standard form (1.2). An overview of integrable autonomous cases and solutions of some nonintegrable PDE models are given. Section 6 deals with the $2 D$ nonlinear Schrödinger equations. Applications to varying inhomogeneous media including fiber optics, ionospheric plasma, hydrodynamics, crystal film channeling, theory of open cavities, and Bose condensation will be discussed elsewhere.

\section{Nonlinear PARABOlic EQUATION}

The propagation of electromagnetic waves in a medium, like ionospheric plasma, can be described by the phenomenological Maxwell equations [77. The corresponding electric field satisfies

$$
\nabla^{2} \boldsymbol{E}-\frac{4 \pi \mu}{c^{2}} \frac{\partial}{\partial t}(\widehat{\sigma} \boldsymbol{E})-\frac{\mu}{c^{2}} \frac{\partial^{2}}{\partial t^{2}}(\widehat{\varepsilon} \boldsymbol{E})=\nabla(\nabla \cdot \boldsymbol{E})-\frac{\nabla \mu}{\mu} \times(\nabla \times \boldsymbol{E}),
$$

where $\widehat{\sigma}$ and $\widehat{\varepsilon}$ are operators of the conductivity and of the electric permittivity of plasma [37]. In what follows, the magnetic permeability $\mu$ of the nonionized medium is equal to unity. (In general, this inhomogeneous equation is highly nonlinear since the tensors $\widehat{\sigma}$ and $\widehat{\varepsilon}$ depend on the field magnitude $|\boldsymbol{E}|$ and the spacial variable $\boldsymbol{r}$.) When the wave frequency $\omega$ is large enough and the time $1 / \omega$ is much less than the characteristic time of establishing the average temperature of the electrons in plasma, one may use successive approximations in powers of the small parameter $\delta \nu_{\mathrm{e}} / \omega$. Assuming that the conductivity $\widehat{\sigma}$ and the electric permittivity $\widehat{\varepsilon}$ are 
time-independent in the first-order approximation, one obtains the familiar nonlinear wave equation [37, [77]:

$$
\Delta \boldsymbol{E}^{(1)}-\operatorname{grad} \operatorname{div} \boldsymbol{E}^{(1)}+\frac{\omega^{2}}{c^{2}} \widehat{\epsilon} \boldsymbol{E}^{(1)}=0, \quad \widehat{\epsilon}=\widehat{\varepsilon}^{(1)}+4 \pi i \widehat{\sigma}^{(1)} / \omega .
$$

In this approximation, $\Lambda\left(e^{-i \omega t} f(\boldsymbol{r})\right)=e^{-i \omega t} \Lambda f(\boldsymbol{r})$ for an operator $\Lambda=\left\{\widehat{\varepsilon}^{(1)}, \widehat{\sigma}^{(1)}\right\}$ and a wave arriving at the plasma boundary preserves its frequency $\omega$ [37.

Equation (2.2) can be significantly simplified when the properties of the medium vary slowly over the wave length and the paraxial approximation is used. In the ionosphere, this is valid at $\omega^{2} \gg \omega_{H}^{2}=(e H / m c)^{2}$ (Larmor frequency), when the complex dielectric tensor is a scalar and can be represented as follows:

$$
\widehat{\epsilon}=\varepsilon_{0}(s)+\widehat{\varepsilon}_{n}\left(\boldsymbol{r}, i^{-1} \partial / \partial \boldsymbol{r}, \boldsymbol{E}\right)+i \varepsilon_{1} .
$$

Here, $\varepsilon_{0}$ and $\varepsilon_{1}=4 \pi \sigma_{0} / \omega$ are the real and imaginary parts of the dielectric constant of inhomogeneous medium, while the operator $\widehat{\varepsilon}_{n}$ depends on the physical mechanism of the nonlinearity [8, 37.

Upon assumption that the operator $\widehat{\epsilon}$ is not essentially altered over the wave length by the inhomogeneity of the plasma and by the nonlinear effect, the electric field can be written as

$$
\boldsymbol{E}^{(1)}=e E(\boldsymbol{r}) \exp \left(i \int_{0}^{s} k\left(s^{\prime}\right) d s^{\prime}\right), \quad k=\frac{\omega}{c} \sqrt{\varepsilon_{0}(s)},
$$

where the field amplitude changes much slower than the phase. (Here, $k$ is the modulus of the wave vector in the linear approximation and $\boldsymbol{e}$ is the unit polarization vector, which is assumed to be a constant [30, 37]). As a result, the propagation of a wave of frequency $\omega$ in plasma, with self-action taken into account, is described by the nonlinear parabolic equation [37]:

$$
\Delta_{\perp} E+2 i k \frac{\partial E}{\partial s}+i E \frac{d k}{d s}+\frac{\omega^{2}}{c^{2}}\left(\widehat{\varepsilon}_{n}+i \varepsilon_{1}\right) E=0 \quad\left(\left|\frac{\partial^{2} E}{\partial s^{2}}\right| \ll \Delta_{\perp} E\right),
$$

where $\Delta_{\perp}$ is the Laplace operator in the plane perpendicular to the beam propagation direction $s$ (see [8], 27, 37] for more details on the derivation of parabolic equation (2.5) from Maxwell's equations; see also [8], 62] for a generic case of turbulent medium and [6], 7], 30], 40], 41, 73], 78, and the references therein for applications of the parabolic equation in nonlinear optics, theory of open cavities and waveguides, plasma physics, acoustics and hydrodynamics). In the case of plane wave $\left(\Delta_{\perp} E=0\right)$ and in the absence of nonlinearity $\left(\widehat{\varepsilon}_{n}=0\right)$ or of inhomogeneity $(d k / d s=0)$, the solution of parabolic equation is well-known [30, 37.

In this paper, we consider an important in practice case of plane-layered medium, when the properties of plasma may vary in one direction, say $s=z$ with $\widehat{\sigma}=$ $Q\left(z, \boldsymbol{r}_{\perp}, i^{-1} \partial / \partial \boldsymbol{r}_{\perp}\right)$ and $\widehat{\varepsilon}=P\left(z, \boldsymbol{r}_{\perp}, i^{-1} \partial / \partial \boldsymbol{r}_{\perp}\right)$ being two quadratic forms of the variables $\boldsymbol{r}_{\perp}$ in perpendicular direction and its gradient $\nabla_{\perp}$ at any $z$ under consideration. The wave propagates in the $z$ direction and the boundary condition is given by $\left.E\right|_{z=0}=E_{0}\left(\boldsymbol{r}_{\perp}\right)$. In the linear case, this wave propagation in geometrical optics is mathematically equivalent to the problem of two-dimensional generalized driven harmonic oscillator in quantum mechanics (see, for example, 27]). The intrinsic connection between Hamiltonian mechanics and the process of wave propagation is anything but a new idea [30, [78. Yet, in view of this analogy between optics and quantum mechanics, the diffraction model under consideration is integrable in terms of the quadratures. The linear parabolic equation (2.5) can be thought of as 
the time-dependent Schrödinger equation. (The $z$ coordinate in the direction of the wave propagation plays a role of time with the perpendicular variables $\boldsymbol{r}_{\perp}$ being two spatial coordinates [27].)

The $2 D$ wave equation in paraxial optics, which is of interest here, can be studied by separation of the variables in normal coordinates. The corresponding initial value problem for the Schrödinger equation, and therefore the boundary values problem for the original parabolic equation, can be solved with the help of the Green function/Feynman propagator or by eigenfunction expansion (see, for example, 22, [49], 50, 70] and the references therein regarding the $1 D$ spatial case). Solution in terms of Airy functions [30] found in [53] are also generalized. The corresponding nearly singular solutions are related to strong scintillation regimes of wave propagation in inhomogeneous media. After discussion of these important examples from paraxial optics, explicit transformations of the nonlinear inhomogeneous parabolic equations into corresponding homogeneous forms will be analyzed.

\section{3. $1 D$ INHOMOGENEOUS MEDIA IN QUADRATIC APPROXIMATION}

In the linear case, when $\widehat{\varepsilon}_{n}$ is a quadratic function of spatial variables and of their gradient in the direction perpendicular to the wave propagation, equation (2.5) is integrable in quadratures (see, for example, 49] and the references therein). The average beam trajectory can be described by the corresponding Ehrenfest theorem thus stressing the analogy between geometrical optics and classical mechanics. Solution of the corresponding boundary value problem can be found in terms the Fresnel integral (or Green's function). In the context of quantum mechanics, the $1 D$ linear Schrödinger equation, which describes the most general quadratic model of this kind,

$$
i \psi_{t}=-a(t) \psi_{x x}+b(t) x^{2} \psi-i c(t) x \psi_{x}-i d(t) \psi-f(t) x \psi+i g(t) \psi_{x}
$$

$(a, b, c, d, f$, and $g$ are suitable real-valued functions of time only), can be solved (formally) by the integral superposition principle:

$$
\psi(x, t)=\int_{-\infty}^{\infty} G(x, y, t) \psi(y, 0) d y,
$$

where

$$
\begin{aligned}
& G(x, y, t)=\left(2 \pi \mu_{0}(t)\right)^{-1 / 2} \\
& \quad \times \exp \left[i\left(\alpha_{0}(t) x^{2}+\beta_{0}(t) x y+\gamma_{0}(t) y^{2}+\delta_{0}(t) x+\varepsilon_{0}(t) y+\kappa_{0}(t)\right)\right],
\end{aligned}
$$

for suitable initial data $\psi(x, 0)=\varphi(x)$ (see [22, [70] and the references therein for more details). In paraxial optics, when the time variable $t$ represents the coordinate, say $z$, in the direction of wave propagation, expressions (3.2)-(3.3) are known as the Fresnel integral [3], 77].

The coefficients $\alpha_{0}, \beta_{0}, \gamma_{0}, \delta_{0}, \varepsilon_{0}, \kappa_{0}$ are given by [22, [70]:

$$
\begin{aligned}
& \alpha_{0}(t)=\frac{1}{4 a(t)} \frac{\mu_{0}^{\prime}(t)}{\mu_{0}(t)}-\frac{d(t)}{2 a(t)}, \\
& \beta_{0}(t)=-\frac{\lambda(t)}{\mu_{0}(t)}, \quad \lambda(t)=\exp \left(-\int_{0}^{t}(c(s)-2 d(s)) d s\right), \\
& \gamma_{0}(t)=\frac{1}{2 \mu_{1}(0)} \frac{\mu_{1}(t)}{\mu_{0}(t)}+\frac{d(0)}{2 a(0)}
\end{aligned}
$$


and

$$
\begin{aligned}
\delta_{0}(t)= & \frac{\lambda(t)}{\mu_{0}(t)} \int_{0}^{t}\left[\left(f(s)-\frac{d(s)}{a(s)} g(s)\right) \mu_{0}(s)+\frac{g(s)}{2 a(s)} \mu_{0}^{\prime}(s)\right] \frac{d s}{\lambda(s)} \\
\varepsilon_{0}(t)= & -\frac{2 a(t) \lambda(t)}{\mu_{0}^{\prime}(t)} \delta_{0}(t)+8 \int_{0}^{t} \frac{a(s) \sigma(s) \lambda(s)}{\left(\mu_{0}^{\prime}(s)\right)^{2}}\left(\mu_{0}(s) \delta_{0}(s)\right) d s \\
& +2 \int_{0}^{t} \frac{a(s) \lambda(s)}{\mu_{0}^{\prime}(s)}\left(f(s)-\frac{d(s)}{a(s)} g(s)\right) d s \\
\kappa_{0}(t)= & \frac{a(t) \mu_{0}(t)}{\mu_{0}^{\prime}(t)} \delta_{0}^{2}(t)-4 \int_{0}^{t} \frac{a(s) \sigma(s)}{\left(\mu_{0}^{\prime}(s)\right)^{2}}\left(\mu_{0}(s) \delta_{0}(s)\right)^{2} d s \\
& -2 \int_{0}^{t} \frac{a(s)}{\mu_{0}^{\prime}(s)}\left(\mu_{0}(s) \delta_{0}(s)\right)\left(f(s)-\frac{d(s)}{a(s)} g(s)\right) d s
\end{aligned}
$$

$\left(\delta_{0}(0)=-\varepsilon_{0}(0)=g(0) /(2 a(0))\right.$ and $\left.\kappa_{0}(0)=0\right)$ provided that $\mu_{0}$ and $\mu_{1}$ are the standard (real-valued) solutions of the characteristic equation:

$$
\mu^{\prime \prime}-\tau(t) \mu^{\prime}+4 \sigma(t) \mu=0
$$

with varying coefficients

$$
\tau(t)=\frac{a^{\prime}}{a}-2 c+4 d, \quad \sigma(t)=a b-c d+d^{2}+\frac{d}{2}\left(\frac{a^{\prime}}{a}-\frac{d^{\prime}}{d}\right),
$$

subject to the initial conditions $\mu_{0}(0)=0, \mu_{0}^{\prime}(0)=2 a(0) \neq 0$ and $\mu_{1}(0) \neq 0$, $\mu_{1}^{\prime}(0)=0$. Here, for applications to turbulent ionospheric plasma (62], 72]), the integrals are treated in the most general way which includes stochastic calculus; see, for example, 28, 61.

An important particular solution (generalized Gaussian-Hermite beams in optics) is given by [49]:

$$
\psi_{n}(x, t)=\frac{e^{i\left(\alpha x^{2}+\delta x+\kappa\right)+i(2 n+1) \gamma}}{\sqrt{2^{n} n ! \mu \sqrt{\pi}}} e^{-(\beta x+\varepsilon)^{2} / 2} H_{n}(\beta x+\varepsilon),
$$

where $H_{n}(x)$ are the Hermite polynomials [59]. Here,

$$
\begin{aligned}
& \mu=\mu(0) \mu_{0} \sqrt{\beta^{4}(0)+4\left(\alpha(0)+\gamma_{0}\right)^{2}}, \\
& \alpha=\alpha_{0}-\beta_{0}^{2} \frac{\alpha(0)+\gamma_{0}}{\beta^{4}(0)+4\left(\alpha(0)+\gamma_{0}\right)^{2}}, \\
& \beta=-\frac{\beta(0) \beta_{0}}{\sqrt{\beta^{4}(0)+4\left(\alpha(0)+\gamma_{0}\right)^{2}}}=\frac{\beta(0) \mu(0)}{\mu(t)} \lambda(t), \\
& \gamma=\gamma(0)-\frac{1}{2} \arctan \frac{\beta^{2}(0)}{2\left(\alpha(0)+\gamma_{0}\right)}, \quad a(0)>0
\end{aligned}
$$


and

$$
\begin{aligned}
\delta= & \delta_{0}-\beta_{0} \frac{\varepsilon(0) \beta^{3}(0)+2\left(\alpha(0)+\gamma_{0}\right)\left(\delta(0)+\varepsilon_{0}\right)}{\beta^{4}(0)+4\left(\alpha(0)+\gamma_{0}\right)^{2}}, \\
\varepsilon= & \frac{2 \varepsilon(0)\left(\alpha(0)+\gamma_{0}\right)-\beta(0)\left(\delta(0)+\varepsilon_{0}\right)}{\sqrt{\beta^{4}(0)+4\left(\alpha(0)+\gamma_{0}\right)^{2}}}, \\
\kappa= & \kappa(0)+\kappa_{0}-\varepsilon(0) \beta^{3}(0) \frac{\delta(0)+\varepsilon_{0}}{\beta^{4}(0)+4\left(\alpha(0)+\gamma_{0}\right)^{2}} \\
& +\left(\alpha(0)+\gamma_{0}\right) \frac{\varepsilon^{2}(0) \beta^{2}(0)-\left(\delta(0)+\varepsilon_{0}\right)^{2}}{\beta^{4}(0)+4\left(\alpha(0)+\gamma_{0}\right)^{2}}
\end{aligned}
$$

in terms of the fundamental solution (3.4) - 3.9) subject to the arbitrary initial data $\mu(0) \neq 0, \alpha(0), \beta(0) \neq 0, \gamma(0), \delta(0), \varepsilon(0), \kappa(0)$. (Equations (3.14)-(3.19) solve the Ermakov-type system (5.7) -(5.12) below with $c_{0}=1$. The complex form of these solutions is found in [46.)

By the superposition principle, solutions (3.12) can be used for the corresponding eigenvalue expansions. In our approach, functions $f$ and $g$ are treated as two stochastic processes and equations (3.7)-(3.9) and (3.17) $-(3.19)$ can be analyzed by statistical methods [5], 62] (which may include random initial data).

A solution in terms of Airy function [30] (generalized Airy beams) has the form:

$$
\psi(x, t)=\frac{e^{i\left(\alpha x^{2}+\delta x+\kappa\right)-i\left(\beta x+\varepsilon-2 \gamma^{2} / 3\right) \gamma}}{\sqrt{\mu}} \operatorname{Ai}\left(\beta x+\varepsilon-\gamma^{2}\right),
$$

where

$$
\begin{aligned}
& \mu(t)=2 \mu(0) \mu_{0}(t)\left(\alpha(0)+\gamma_{0}(t)\right), \\
& \alpha(t)=\alpha_{0}(t)-\frac{\beta_{0}^{2}(t)}{4\left(\alpha(0)+\gamma_{0}(t)\right)}, \\
& \beta(t)=-\frac{\beta(0) \beta_{0}(t)}{2\left(\alpha(0)+\gamma_{0}(t)\right)}=\frac{\beta(0) \mu(0)}{\mu(t)} \lambda(t), \\
& \gamma(t)=\gamma(0)-\frac{\beta^{2}(0)}{4\left(\alpha(0)+\gamma_{0}(t)\right)}
\end{aligned}
$$

and

$$
\begin{aligned}
\delta(t) & =\delta_{0}(t)-\frac{\beta_{0}(t)\left(\delta(0)+\varepsilon_{0}(t)\right)}{2\left(\alpha(0)+\gamma_{0}(t)\right)}, \\
\varepsilon(t) & =\varepsilon(0)-\frac{\beta(0)\left(\delta(0)+\varepsilon_{0}(t)\right)}{2\left(\alpha(0)+\gamma_{0}(t)\right)}, \\
\kappa(t) & =\kappa(0)+\kappa_{0}(t)-\frac{\left(\delta(0)+\varepsilon_{0}(t)\right)^{2}}{4\left(\alpha(0)+\gamma_{0}(t)\right)} .
\end{aligned}
$$

(These equations solve the Riccati-type system (5.7)-(5.12) with $c_{0}=0$.) It is worth noting that our solution resembles the main features, in the linear approximation, of rogue waves 44, [53, 68. Similar solutions can be obtained with the help of Airy beams found in [9], 66], and 67] (see [53] and the references therein). Once again, functions $f$ and $g$ may be treated as stochastic processes. 
Examples. Use of (3.19)-(3.25) from [50] in equation (3.12) provides a new family of oscillating Gaussian-Hermite beams in $1 D$ homogeneous medium (see also [4, [5], 77] and the next section for an extension to the $2 D$ case). The "missing" solutions for the simple harmonic oscillator [51] provide new examples of oscillating Gaussian-Hermite beams in parabolic (self-focusing fiber) waveguides discussed in [4, 30, 52, 78. Particular solutions in terms of Airy functions for the planelayered medium can be obtained in analogy with [53] and [30]. All of these solutions are derived with the help of Theorem 1 , where the simplest possible nonlinear terms are also analyzed.

\section{4. $2 D$ INHOMOGENEOUS MEDIA IN QUADRATIC APPROXIMATION}

The Schrödinger (parabolic) equation is given by

$$
i \psi_{t}(\boldsymbol{r}, t)=H \psi(\boldsymbol{r}, t), \quad H=H_{1}(x)+H_{2}(y),
$$

where $H_{1,2}$ are the Hamiltonians in $x$ and $y$ directions similar to one in (3.1) but, in general, with two different sets of suitable functions $a_{1,2}(t), b_{1,2}(t), c_{1,2}(t)$, $d_{1,2}(t), f_{1,2}(t)$ and $g_{1,2}(t)$. (We assume that the nondiagonal terms are eliminated by passing to normal coordinates.) The Green function can be obtained as the product:

$$
G\left(\boldsymbol{r}, \boldsymbol{r}^{\prime}, t\right)=G_{1}(x, \xi, t) G_{2}(y, \eta, t),
$$

where the kernels $G_{1,2}$ are given by (3.3) with a simple change of notation: The coefficients $\alpha_{0}^{(1,2)}(t), \beta_{0}^{(1,2)}(t), \gamma_{0}^{(1,2)}(t), \delta_{0}^{(1,2)}(t), \varepsilon_{0}^{(1,2)}(t), \kappa_{0}^{(1,2)}(t)$ are defined, in general, in terms of two sets of the fundamental solutions (3.4)-(3.9).

Solution of the corresponding boundary-values problem can be found by the integral superposition principle (or generalized Fresnel integral):

$$
\psi(\boldsymbol{r}, t)=\int_{\boldsymbol{R}^{2}} G\left(\boldsymbol{r}, \boldsymbol{r}^{\prime}, t\right) \psi\left(\boldsymbol{r}^{\prime}, t=0\right) d \boldsymbol{r}^{\prime}
$$

for suitable initial data.

The $2 D$ generalized Gaussian-Hermite beams have the form

$$
\begin{aligned}
\psi_{n m} & (\boldsymbol{r}, t)=\frac{e^{i\left(\kappa_{1}+\kappa_{2}\right)}}{\sqrt{2^{n+m} n ! m ! \mu^{(1)} \mu^{(2)} \pi}} \\
& \times e^{i\left(\alpha_{1} x^{2}+\delta_{1} x\right)+i(2 n+1) \gamma_{1}} e^{i\left(\alpha_{2} y^{2}+\delta_{2} y\right)+i(2 m+1) \gamma_{2}} \\
& \times e^{-\left(\beta_{1} x+\varepsilon_{1}\right)^{2} / 2-\left(\beta_{2} y+\varepsilon_{2}\right)^{2} / 2} H_{n}\left(\beta_{1} x+\varepsilon_{1}\right) H_{m}\left(\beta_{2} y+\varepsilon_{2}\right) .
\end{aligned}
$$

Equations (3.13) - 3.19) are valid with a similar change of notation for given initial data $\mu^{(1,2)}(0), \alpha_{1,2}(0), \beta_{1,2}(0) \neq 0, \gamma_{1,2}(0), \delta_{1,2}(0), \varepsilon_{1,2}(0), \kappa_{1,2}(0)$ (see also 4, 5, 77 for important special cases). In general, the product of any two $1 D$ solutions (3.12) and (3.20), say

$$
\psi_{n}(\boldsymbol{r}, t)=\psi_{n}(x, t) \psi(y, t),
$$

gives an important class of $2 D$ solutions (Airy-Gaussian-Hermite beams in inhomogeneous media).

Example: Spiral Laser Beams in Varying Media. In the inhomogeneous $2 D$ case under consideration, equation (4.1) can be reduced to the standard forms

$$
-i \chi_{\tau}+\chi_{\xi \xi}+\chi_{\eta \eta}=c_{0}\left(\xi^{2}+\eta^{2}\right) \chi \quad\left(c_{0}=0,1\right)
$$


(see Lemma 1 with $h_{0}=0$; we have assumed that $a_{1} \beta_{1}^{2}=a_{2} \beta_{2}^{2}[23]$ ). By the Ansatz $\Psi(X, Y, T)=\chi(\xi, \eta, \tau), T=-\tau$ and

$$
\left(\begin{array}{l}
X \\
Y
\end{array}\right)=\left(\begin{array}{cc}
\cos \omega \tau & -\sin \omega \tau \\
\sin \omega \tau & \cos \omega \tau
\end{array}\right)\left(\begin{array}{l}
\xi \\
\eta
\end{array}\right), \quad \omega=\text { constant }
$$

equation (4.6) with $c_{0}=1$ can be transformed to an equation of motion for the isotropic planar harmonic oscillator in a perpendicular uniform magnetic field:

$$
i \Psi_{T}+\Psi_{X X}+\Psi_{Y Y}=i \omega\left(X \Psi_{Y}-Y \Psi_{X}\right)+\left(X^{2}+Y^{2}\right) \Psi .
$$

The latter was solved in polar coordinates $X=R \cos \Theta, Y=R \sin \Theta$ (as an example of a simple degenerated unperturbed system) by Fock in the early days of quantum mechanics 29]:

$$
\begin{gathered}
\Psi(R, \Theta, T)=\sqrt{\frac{n !}{\pi(n+|m|) !}} e^{-i E T} e^{i m \Theta} R^{|m|} e^{-R^{2} / 2} L_{n}^{|m|}\left(R^{2}\right), \\
E=4 n+2(|m|+1)-m \omega \quad(m= \pm 0, \pm 1, \ldots n=0,1, \ldots)
\end{gathered}
$$

in terms of Laguerre polynomials [59]. (This wave function coincides, up to a simple factor, with the one for a flat isotropic oscillator without magnetic field. Therefore, its development in terms of (4.4) for standard harmonics is a $2 D$ special case of the multidimensional expansions from [59].) By back substitution, one arrives at a general family of rotating solutions. Numerous examples of the spiral laser beams in the uniform medium are discussed in [3] (see also [4], [5], 77]). Similar to [50, one gets a multi-parameter family of solutions in a $2 D$ medium [52].

A similar effect of the superfocusing of a proton beam in a thin monocrystal film was discussed in [25], 26] (the validity of the $2 D$ harmonic crystal model had been confirmed through Monte Carlo computer experiments).

\section{Transformation of Related $1 D$ nOnlinear Equations}

In this section, we complexify all time-dependent coefficients of the linear equation (3.1) and analyze the simplest nonautonomous nonlinear terms. For a generic nonautonomous (derivative) nonlinear Schrödinger equation of the form

$$
i \psi_{t}=H \psi+R(\psi),
$$

where $H$ is an arbitrary varying quadratic Hamiltonian and $R(\psi)=P\left(\psi, \psi^{*}, \psi_{x}, \psi_{x}^{*}\right)$ is a polynomial in four variables, we assume the natural gauge invariance condition:

$$
P\left(\psi e^{i S}, \psi^{*} e^{-i S},\left(\psi e^{i S}\right)_{x},\left(\psi^{*} e^{-i S}\right)_{x}\right)=C e^{i S} P\left(\psi, \psi^{*}, \psi_{x}, \psi_{x}^{*}\right)
$$

(also known as $U(1)$, or phase invariance). The lowest terms that satisfy this condition are given by

$$
P\left(\psi, \psi^{*}, \psi_{x}, \psi_{x}^{*}\right)=h_{0} \psi+\left(h_{1} x+h_{2}\right)|\psi|^{2} \psi+i h_{3}|\psi|^{2} \psi_{x}+i h_{4} \psi^{2} \psi_{x}^{*}+h_{5}|\psi|^{4} \psi,
$$

where $h_{k}=h_{k}(x, t), k=0, \ldots, 5$ are some real or complex-valued functions. Our result is the following.

Theorem 1. The substitution

$$
\psi=\frac{e^{i\left(\alpha(t) x^{2}+\delta(t) x+\kappa(t)\right)}}{\sqrt{\mu(t)}} \chi(\xi, \tau), \quad \xi=\beta(t) x+\varepsilon(t), \quad \tau=\gamma(t)
$$


transforms the nonautonomous and inhomogeneous equation (5.1) with the lowest gauge invariant nonlinearities (5.3), namely,

$$
\begin{gathered}
i \psi_{t}+a(t) \psi_{x x}-b(t) x^{2} \psi+i c(t) x \psi_{x}+i d(t) \psi+f(t) x \psi-i g(t) \psi_{x} \\
=h_{0} \psi+\left(h_{1} x+h_{2}\right)|\psi|^{2} \psi+i h_{3}|\psi|^{2} \psi_{x}+i h_{4} \psi^{2} \psi_{x}^{*}+h_{5}|\psi|^{4} \psi,
\end{gathered}
$$

into the autonomous form

$$
\begin{aligned}
& -i \chi_{\tau}+\chi_{\xi \xi}-c_{0} \xi^{2} \chi \\
& =d_{0} \chi+\left(d_{1} \xi+d_{2}\right)|\chi|^{2} \chi+i d_{3}|\chi|^{2} \chi_{\xi}+i d_{4} \chi^{2}\left(\chi_{\xi}\right)^{*}+d_{5}|\chi|^{4} \chi
\end{aligned}
$$

$\left(c_{0}=0,1\right)$ provided that

$$
\frac{d \alpha}{d t}+b+2 c \alpha+4 a \alpha^{2}=c_{0} a \beta^{4}
$$

$$
\frac{d \beta}{d t}+(c+4 a \alpha) \beta=0
$$

$$
\frac{d \gamma}{d t}+a \beta^{2}=0
$$

and

$$
\begin{gathered}
\frac{d \delta}{d t}+(c+4 a \alpha) \delta=f+2 g \alpha+2 c_{0} a \beta^{3} \varepsilon, \\
\frac{d \varepsilon}{d t}=(g-2 a \delta) \beta \\
\frac{d \kappa}{d t}=g \delta-a \delta^{2}+c_{0} a \beta^{2} \varepsilon^{2} .
\end{gathered}
$$

Here

$$
\alpha=\frac{1}{4 a} \frac{\mu^{\prime}}{\mu}-\frac{d}{2 a}
$$

and

$$
\begin{aligned}
& h_{1}=a \beta^{2}|\mu|\left(d_{1} \beta+\frac{2 \alpha}{\beta} d_{3}-\frac{2 \alpha^{*}}{\beta^{*}} d_{4}\right) e^{2 \operatorname{Im} S}, \\
& h_{2}=a \beta^{2}|\mu|\left[d_{1} \varepsilon+d_{2}+\frac{\delta}{\beta} d_{3}-\frac{\delta^{*}}{\beta^{*}} d_{4}\right] e^{2 \operatorname{Im} S}, \\
& h_{3}=d_{3} a \beta|\mu| e^{2 \operatorname{Im} S}, \quad h_{4}=d_{4} a \frac{\beta^{2}}{\beta^{*}}|\mu| e^{2 \operatorname{Im} S}, \\
& h_{5}=d_{5} a \beta^{2}|\mu|^{2} e^{4 \operatorname{Im} S}, \quad h_{0}=d_{0} a \beta^{2}
\end{aligned}
$$

$\left(d_{0}, d_{1}, d_{2}, d_{3}, d_{4}, d_{5}\right.$ are constants and $\left.S=\alpha x^{2}+\delta x+\kappa\right)$.

Proof. For the case of complex-valued coefficients, the transformation of the linear part is similar to [49] and 70] (with the use of contour integration if needed). 
Changing the variables in the nonlinear part,

$$
\begin{aligned}
& \mu^{1 / 2} e^{-i S} P=h_{0} \chi+i h_{3} \frac{\beta}{|\mu|} e^{-2 \operatorname{Im} S}|\chi|^{2} \chi_{\xi}+i h_{4} \frac{\beta^{*}}{|\mu|} e^{-2 \operatorname{Im} S} \chi^{2}\left(\chi_{\xi}\right)^{*} \\
& +\frac{h_{5}}{|\mu|^{2}} e^{-4 \operatorname{Im} S}|\chi|^{4} \chi \\
& +\left[h_{2}-h_{3} \delta+h_{4} \delta^{*}+\left(h_{1}-2 \alpha h_{3}+2 \alpha^{*} h_{4}\right) \frac{\xi-\varepsilon}{\beta}\right] e^{-2 \operatorname{Im} S \frac{|\chi|^{2}}{|\mu|} \chi}
\end{aligned}
$$

and substituting into (5.5), one completes the proof with the aid of our conditions (5.14) - (5.17)

Remark 1. The systems (5.7)-(5.12) can be solved with the help of variants of a nonlinear superposition principle established in 49] and [69]; see equations (3.22)(3.27) and (3.14) -(3.19) for $c_{0}=0,1$, respectively.

The autonomous cubic nonlinear Schrödinger equation is completely integrable by the inverse scattering method [1, [2, 60, [81. When $c_{0}=d_{0}=d_{1}=d_{3}=$ $d_{4}=d_{5}=0, d_{2}=h_{0} \neq 0$ and $d_{0}=d_{1}=d_{2}=d_{3}=d_{4}=0, d_{5}=h_{0} \neq 0$, we reproduce the results of [69] and [70] (for $p=2$ and $p=4$, respectively), where the nonautonomous integrability condition was established (see also the references therein). The derivative nonlinear Schrödinger equation, which describes the propagation of circular polarized nonlinear Alfvén waves in plasma physics [56, 57, [79], arises when $c_{0}=d_{0}=d_{1}=d_{2}=d_{5}=0$ and $d_{3}=2 d_{4}$ (see, for example, [7, [8, 10, 17] and the references therein for methods of solution of this equation; our theorem identifies the corresponding nonautonomous integrable model). The amplitude equations of cubic-quintic type have been derived via asymptotic analysis of the governing equations of fluid mechanics near the onset of instability [40, 44]. Among other special cases are the Chen-Lee-Lui derivative nonlinear Schrödinger equation [10] and the Gerdjikov-Ivanov equation. Generic autonomous equations of the type (5.6) and some of their extensions such as quintic complex GinzburgLandau equation are discussed, for example, in [14, [15], 17, [20, 21, 31, 32, [33, 34], 35, 40], 43], 47, 55], 63, 64, 80, (see also the references therein; an extensive bibliography will be given elsewhere).

In the case $c_{0}=d_{0}=d_{1}=0$, a detailed Painlevé analysis of equation (5.6) is performed by Clarkson and Cosgrove [17] (see also [15] for the extension to the case of complex parameters). They have shown that this equation possesses the Painlevé property for partial differential equations only if $d_{5}=d_{4}\left(2 d_{4}-d_{3}\right) / 4$. When this relation holds, the latter is equivalent under a gauge transformation [43], [47]:

$$
\chi=\phi \exp \left(-i \nu \int_{\xi_{0}}^{\xi}|\phi|^{2} d \eta\right),
$$

to a hybrid of the nonlinear Schrödinger equation and the derivative nonlinear Schrödinger equation [7, 8],

$$
-i \phi_{\tau}+\phi_{\xi \xi}+\lambda|\phi|^{2} \phi+i \mu\left(|\phi|^{2} \phi\right)_{\xi}=0
$$

( $\lambda, \mu$ and $\nu$ are constants), where one can assume that $\lambda=0$ without loss of generality [80]. The corresponding derivative nonlinear Schrödinger equation is known to be completely integrable [2, [10] (a detailed bibliography will be provided 
elsewhere). Then solutions of the original equation are constructed by the gauge transformation (5.19) in principle (see, for example, Refs. [17, [15] and [43] for more details). Explicit solitary wave solutions can be found in [43], [55], 64] (review also the references therein). Theorem 1 allows us to extend these results to a larger class of nonautonomous and inhomogeneous nonlinear Schrödinger equations.

Exact solitary wave solutions of the one-dimensional quintic complex GinzburgLandau equation are obtained in [55, 64. In their notation and terminology,

$$
\frac{\partial A}{\partial t}=\varepsilon A+\left(b_{1}+i c_{1}\right) \frac{\partial^{2} A}{\partial x^{2}}-\left(b_{3}-i c_{3}\right)|A|^{2} A-\left(b_{5}-i c_{5}\right)|A|^{4} A,
$$

where $\varepsilon, b_{1}, c_{1}, b_{3}, c_{3}, b_{3}, c_{3}$ are real constants and the field $A(x, t)$ is complexvalued (see also [13], 15]). These solutions are expressed in terms of hyperbolic functions and include coherent structures with a strong spatial localization such as pulses and fronts, as well as sources and sinks. Equation (5.21) is a one-dimensional model of the large-scale behavior of many nonequilibrium pattern-forming systems (see, for example, [55, 63, 64] and the references therein). A systematic method for obtaining analytic solitary wave solutions of nonintegrable PDEs has been introduced by Conte and Musette [19], [58] and further developed by Hone [38, 39] and Vernov [75], 76] (see also [15] for another approach and [20, [21, 31, 34] for exact solutions). The unique elliptic traveling wave solution of (5.21) is found in [76]. One may extend some of these results to nonautonomous equations.

If conditions (5.14)-(5.17) are not satisfied in a certain application, yet we can find the corresponding coefficients $d_{k}$ as functions of time and spatial variables thus moving the time dependence into the nonlinear part only. Then one may use perturbation methods and/or some parameter control when possible. The Feshbach resonance in Bose-Einstein condensation provides a classical example of such nonlinearity control [24], 42]. For instance, a justification of the so-called local density approximation [45] can be obtained from Theorem 1 with $c_{0}=0$ and $p=2,4$, when a classical motion of the corresponding quadratic system is already taken into account, under the following adiabatic condition:

$$
\frac{d}{d t}\left(\frac{h}{a(t) \beta^{2}(t) \mu^{p / 2}(t)}\right)=\mathrm{o}(1)(\text { or } \ll 1) .
$$

It is worth noting that Theorem 1 can be used for suitable stochastic process coefficients $f$ and $g$ with the corresponding random initial data in (3.7)-(3.9) and (3.17) - (3.19). Ansatz (5.4) transforms a stochastic field PDE (5.5) into the deterministic one with respect to stochastic spacial variable $\xi$. Then a stochastic process solution of the Ermakov-type system (5.7)-(5.12) with the proper averages can be found (see also [5], 8, 62 for an advanced study).

Last but not least, in several critical autonomous cases, Theorem 1 allows us to derive the maximum symmetry groups and the similarity transformations for the corresponding equations (see, for example, [50, 54 and the references therein). These symmetries result in explicit blow up solutions for those critical cases and variations of initial data can be used for a nonlinear stability analysis. 


\section{6. $2 D$ inhomogeneous nonlinear Schrödinger EQUation}

In a similar fashion, we prove the following.

Lemma 1. The inhomogeneous nonlinear Schrödinger equation

$$
\begin{aligned}
i \psi_{t}= & -a\left(\psi_{x x}+\psi_{y y}\right)+b\left(x^{2}+y^{2}\right) \psi-i c\left(x \psi_{x}+y \psi_{y}\right)-2 i d \psi \\
& -\left(x f_{1}+y f_{2}\right) \psi+i\left(g_{1} \psi_{x}+g_{2} \psi_{y}\right)+h|\psi|^{p} \psi,
\end{aligned}
$$

where $a, b, c, d, f_{1,2}$ and $g_{1,2}$ are real-valued functions of $t$, can be reduced to the autonomous form

$$
-i \chi_{\tau}+\chi_{\xi \xi}+\chi_{\eta \eta}=c_{0}\left(\xi^{2}+\eta^{2}\right) \chi+h_{0}|\chi|^{p} \chi \quad\left(c_{0}=0,1\right)
$$

by the following Ansatz:

$$
\psi=\mu^{-1} e^{i\left(\alpha\left(x^{2}+y^{2}\right)+\left(\delta_{1} x+\delta_{2} y\right)+\kappa_{1}+\kappa_{2}\right)} \chi(\xi, \eta, \tau),
$$

where $\xi=\beta x+\varepsilon_{1}, \eta=\beta y+\varepsilon_{2}, \tau=\gamma$, and $h=h_{0} a \beta^{2} \mu^{p} \quad$ ( $h_{0}$ is a constant). Here, solutions of the system (5.7) -(5.12) are given by (3.21) -(3.27) and (3.13)-(3.19) for $c_{0}=0$ and $c_{0}=1$, respectively.

In the $1 D$ linear case, where nonspreading Airy beams were introduced [9] (see also [66], 67]), the symmetry of free Schrödinger equation can be used in order to obtain new exact solutions [53]. Although the corresponding $1 D$ cubic nonlinear Schrödinger equation is no longer preserved under the expansion transformation (but has a similarity reduction to the second Painlevé equation [34, [36, [53, 68], [71]), the same symmetry holds for the quintic nonlinear Schrödinger equation, which is thus invariant under the action of this group. Here, the blow up, namely a singularity such that the wave amplitude tends to infinity in a finite time, occurs (see [54], 70], 74] and the references therein).

As is well known, a similar symmetry holds for the homogeneous $2 D$ cubic nonlinear Schrödinger equation [48, 73. (in optics this symmetry is known as Talanov's transformation [78]). The most general symmetry of this kind follows immediately from our lemma when $p=2$ with the aid of (3.1)-(3.4) of [50]. This is another classical example of blow up phenomenon. The stationary $2 D$ waveguides in homogeneous quadratic Kerr media are unstable [48. Under certain condition, self-focusing of light beams occur on a finite distance despite diffraction spreading. Moreover, for parabolic channels in a monocrystal film, the cubic nonlinearity may further enhance superfocusing of particle beams predicted in [25], 26]. The corresponding inhomogeneous medium effects deserve a detailed study. An extension to randomly varying media is also of interest (cf. [5], 28, 62, 62]).

\section{ACKNOWLEDGMENTS}

The authors thank Robert Conte for important references. The authors are grateful to Mark Faifman for bringing references [25], 26] to their attention and for valuable discussions. The referee's useful suggestions are much appreciated.

\section{REFERENCES}

[1] M. J. Ablowitz and P. A. Clarkson, Solitons, nonlinear evolution equations and inverse scattering, London Mathematical Society Lecture Note Series, vol. 149, Cambridge University Press, Cambridge, 1991. MR.1149378 (93g:35108) 
[2] Mark J. Ablowitz and Harvey Segur, Solitons and the inverse scattering transform, SIAM Studies in Applied Mathematics, vol. 4, Society for Industrial and Applied Mathematics (SIAM), Philadelphia, Pa., 1981. MR642018 (84a:35251)

[3] E. G. Abramochkin and V. G. Volostnikov, Spiral light beams, Physics-Uspekhi 47 (2004), no. $12,1177-1203$.

[4] G. P. Agrawal, A. K. Ghatak, and C. L. Mehtav, Propagation of a partially coherent beam through selfoc fibers, Opt. Comm. 12 (1974), no. 3, 333-337.

[5] S. A. Akhmanov, Yu. E. D'yakov, and A. S. Chirkin, Vvedenie v statisticheskuyu radiofiziku i optiku (Russian), Nauka, Moscow, 1981. MR626992 (83c:78001)

[6] S. A. Akhmanov, A. P. Sukhorukov, and R. V. Khokhlov, Self-focusing and diffraction of light in a nonlinear medium, Physics-Uspekhi 10 (1968), no. 1-2, 609-636.

[7] S. A. Akhmanov, V. A. Vyslouh, and A. S. Chirkin, Self-action of wave packets in a nonlinear medium and femtosecond laser pulse generation, Physics-Uspekhi 29 (1986), no. 7, 642-677.

[8] S. A. Akhmanov, V. A. Vyslouh, and A. S. Chirkin, Optics of Femtosecond Laser Pulses, Nauka, Moscow, 1988 [in Russian].

[9] M. V. Berry and N. L. Balazs, Nonspreading wave packets, Am. J. Phys. 47 (1979), no. 2, 264-267.

[10] H. H. Chen, Y. C. Lee, and C. S. Liu, Integrability of nonlinear Hamiltonian systems by inverse scattering method, Special issue on solitons in physics. Phys. Scripta 20 (1979), no. 34, 490-492, DOI 10.1088/0031-8949/20/3-4/026. MR.544493(81e:35008)

[11] Hsing Hen Chen and Chuan Sheng Liu, Solitons in nonuniform media, Phys. Rev. Lett. 37 (1976), no. 11, 693-697. MR0411488 (53 \#15222)

[12] H.-H. Chen and Ch.-Sh. Liu, Nonlinear wave and soliton propagation in media with arbitrary inhomogeneities, Phys. Fluids 21 (1978), no. 3, 377-380.

[13] S. Roy Choudhury, Modulated amplitude waves in the cubic-quintic GinzburgLandau equation, Math. Comput. Simulation 69 (2005), no. 3-4, 243-256, DOI 10.1016/j.matcom.2005.01.003. MR2152257

[14] P. A. Clarkson, Painlevé analysis of the damped, driven nonlinear Schrödinger equation, Proc. Roy. Soc. Edin., 109A (1988), 109-126. MR0952332 (89k:35200)

[15] Peter A. Clarkson, Dimensional reductions and exact solutions of a generalized nonlinear Schrödinger equation, Nonlinearity 5 (1992), no. 2, 453-472. MR.1158381 (93f:35212)

[16] NIST handbook of mathematical functions, U.S. Department of Commerce National Institute of Standards and Technology, Washington, DC, 2010. Edited by Frank W. J. Olver, Daniel W. Lozier, Ronald F. Boisvert and Charles W. Clark; with 1 CD-ROM (Windows, Macintosh and UNIX). MR2723248 (2012a:33001)

[17] Peter A. Clarkson and Christopher M. Cosgrove, Painlevé analysis of the nonlinear Schrödinger family of equations, J. Phys. A 20 (1987), no. 8, 2003-2024. MR893304 (89c:35136)

[18] Robert Conte and Micheline Musette, Linearity inside nonlinearity: exact solutions to the complex Ginzburg-Landau equation, Phys. D 69 (1993), no. 1-2, 1-17, DOI 10.1016/01672789(93)90177-3. MR 1245653 (94m:35281)

[19] R. Conte and M. Musette, Elliptic general analytic solutions, Stud. Appl. Math. 123 (2009), no. 1, 63-81, DOI 10.1111/j.1467-9590.2009.00447.x. MR2538286 (2010m:35053)

[20] Robert Conte and Tuen-Wai Ng, Meromorphic traveling wave solutions of the complex cubic-quintic Ginzburg-Landau equation, Acta Appl. Math. 122 (2012), 153-166, DOI 10.1007/s10440-012-9734-y. MR2993990

[21] R. Conte and T.-W. Ng, Detection and construction of an elliptic solution of the complex cubic-quintic Ginzburg-Landau equation, Theor. Math. Phys. 172 (2012), no. 2, 1073-1084.

[22] Ricardo Cordero-Soto, Raquel M. Lopez, Erwin Suazo, and Sergei K. Suslov, Propagator of a charged particle with a spin in uniform magnetic and perpendicular electric fields, Lett. Math. Phys. 84 (2008), no. 2-3, 159-178, DOI 10.1007/s11005-008-0239-6. MR2415547 $(2009 \mathrm{~m}: 81055)$

[23] R. Kordero-Soto and S. K. Suslov, Time reversal for modified oscillators (Russian, with Russian summary), Teoret. Mat. Fiz. 162 (2010), no. 3, 345-380, DOI 10.1007/s11232-0100023-5; English transl., Theoret. and Math. Phys. 162 (2010), no. 3, 286-316. MR2682129 (2011h:81100)

[24] F. Dalfovo, S. Giorgini, L. P. Pitaevskii, and S. Stringari, Theory of Bose-Einstein condensation in trapped gases, Rev. Mod. Phys. 71 (1999), 463-512. 
[25] Yu. N. Demkov, Channeling, superfocusing, and nuclear reactions, Phys. Atom. Nuclei, 72 (2009), no. 5, 779-785.

[26] Yu. N. Demkov and J. D. Meyer, A sub-atomic microscope, superfocusing in channeling and close encounter atomic and nuclear reactions, Eur. Phys. J. B 42 (2004), 361-365.

[27] V. V. Dodonov and V. I. Manko, Invariants and correlated states of nonstationary quantum systems (Russian), Trudy Fiz. Inst. Lebedev. 183 (1987), 71-181, 289. MR.937883(89i:81044)

[28] Franco Flandoli and Alex Mahalov, Stochastic three-dimensional rotating Navier-Stokes equations: averaging, convergence and regularity, Arch. Ration. Mech. Anal. 205 (2012), no. 1, 195-237, DOI 10.1007/s00205-012-0507-6. MR2927621

[29] V. A. Fock, Selected works. Quantum mechanics and quantum field theory, edited by L. D. Faddeev, L. A. Khalfin and I. V. Komarov. Chapman \& Hall/CRC, Boca Raton, FL, 2004. MR2083062(2005m:81003)

[30] V. A. Fock, Electromagnetic diffraction and propagation problems, International Series of Monographs on Electromagnetic Waves, Vol. 1, Pergamon Press, Oxford, 1965. MR0205569 (34 \#5396)

[31] L. Gagnon, Exact traveling-wave solutions for optical models based on the nonlinear cubicquintic Schrödinger equation, J. Opt. Soc. Amer. A 6 (1989), no. 9, 1477-1483, DOI 10.1364/JOSAA.6.001477. MR.1015382 (90g:78006)

[32] L. Gagnon, G. Grammaticos, A. Ramani, and P. Winternitz, Lie symmetries of a generalised non-linear Schrödinger equation: III. Reductions to third-order ordinary differential equations, J. Phys. A: Math. Gen. 22 (1989), 499-509. MR0984527(90b:35014)

[33] L. Gagnon and P. Winternitz, Lie symmetries of a generalised nonlinear Schrödinger equation. I. The symmetry group and its subgroups, J. Phys. A 21 (1988), no. 7, 1493-1511. MR 951040 (89g:58220)

[34] L. Gagnon and P. Winternitz, Lie symmetries of a generalised nonlinear Schrödinger equation. II. Exact solutions, J. Phys. A 22 (1989), no. 5, 469-497. MR984526 (90b:35013)

[35] L. Gagnon and P. Winternitz, Symmetry classes of variable coefficient nonlinear Schrödinger equations, J. Phys. A 26 (1993), no. 23, 7061-7076. MR1253895 (95f:35238)

[36] J. A. Giannini and R. I. Joseph, The role of the second Painlevé transcendent in nonlinear optics, Phys. Lett. A 141 (1989), no. 8-9, 417-419, DOI 10.1016/0375-9601(89)90860-8. MR 1030927 (90i:78038)

[37] A. V. Gurevich, Nonlinear phenomena in the ionosphere, Springer-Verlag, Berlin, 1978.

[38] A. N. W. Hone, Painlevé tests, singularity structure and integrability, Lecture Notes in Phys., 767, Springer, Berlin, 2009. MR2867552(2012k:34199)

[39] A. N. W. Hone, Non-existence of elliptic travelling wave solutions of the complex GinzburgLandau equation, Phys. D 205 (2005), no. 1-4, 292-306, DOI 10.1016/j.physd.2004.10.011. MR2167157(2006e:35302)

[40] R. S. Johnson, On the modulation of water waves in the neighbourhood of $k h \approx 1.363$, Proc. Roy. Soc. London Ser. A 357 (1977), no. 1689, 131-141. MR0479013 (57 \#18469)

[41] B. B. Kadomtsev and V. I. Karpman, Nonlinear waves (Russian), Uspehi Fiz. Nauk 103 (1971), 193-232; English transl., Soviet Physics Uspekhi 14 (1971), 40-60. MR0449148 (56 \#7453)

[42] Yu. Kagan, E. L. Surkov and G. V. Shlyapnikov, Evolution and global collapse of trapped Bose condensates under variations of the scattering length, Phys. Rev. Lett. 79 (1997), no. 14, 2604-2607.

[43] Saburo Kakei, Narimasa Sasa, and Junkichi Satsuma, Bilinearization of a generalized derivative nonlinear Schrödinger equation, J. Phys. Soc. Japan 64 (1995), no. 5, 1519-1523, DOI 10.1143/JPSJ.64.1519. MR.1335407 (96a:35195)

[44] Christian Kharif, Efim Pelinovsky, and Alexey Slunyaev, Rogue waves in the ocean, Advances in Geophysical and Environmental Mechanics and Mathematics, Springer-Verlag, Berlin, 2009. MR2841079 (2012g:86006)

[45] E. B. Kolomeisky, T. J. Newman, J. P. Straley, and X. Qi, Low-dimensional Bose liquids: beyond the Gross-Pitaevskii approximation, Phys. Rev. Lett. 85 (2000), no. 6, 1146-1149.

[46] C. Krattenthaler, S. I. Kryuchkov, A. Mahalov, and S. K. Suslov, On the problem of electromagnetic-field quantization, arXiv:1301.7328v2 [math-ph] 9 Apr 2013.

[47] A. Kundu, Landau-Lifshitz and higher-order nonlinear systems gauge generated from nonlinear Schrödinger type equations, J. Math. Phys. 25 (1984), no. 12, 3433-3438, DOI 10.1063/1.526113. MR767547 (86c:58069) 
[48] E. A. Kuznetsov and S. K. Turitsyn, Talanov transformations in self-focusing problems and instability of stationary waveguides, Phys. Lett. A 112 (1985), no. 6-7, 273-275.

[49] N. Lanfear, R. M. López and S. K. Suslov, Exact wave functions for generalized harmonic oscillators, J. Russ. Laser Res. 32 (2011), no. 4, 352-361.

[50] R. M. López, S. K. Suslov, and J. M. Vega-Guzmán, Reconstructing the Schrödinger groups, Phys. Scr. 87 (2013), no. 3, 038118 (6 pages).

[51] Raquel M. López, Sergei K. Suslov, and José M. Vega-Guzmán, On a hidden symmetry of quantum harmonic oscillators, J. Difference Equ. Appl. 19 (2013), no. 4, 543-554, DOI 10.1080/10236198.2012.658384. MR3040814

[52] A. Mahalov, E. Suazo, and S. K. Suslov, Spiral laser beams in inhomogeneous media, Opt. Lett. 38 (2013), no. 15, 1-4.

[53] A. Mahalov and S. K. Suslov, An "Airy gun": Self-accelerating solutions of the timedependent Schrödinger equation in vacuum, Phys. Lett. A 377 (2012), 33-38.

[54] A. Mahalov and S. K. Suslov, Wigner function approach to oscillating solutions of the $1 D$ quintic nonlinear Schrödinger equation, J. Nonlinear Opt. Phys. \& Mat. 22 (2013), no. 2, 1350013 (14 pages).

[55] Philippe Marcq, Hugues Chaté, and Robert Conte, Exact solutions of the one-dimensional quintic complex Ginzburg-Landau equation, Phys. D 73 (1994), no. 4, 305-317, DOI 10.1016/0167-2789(94)90102-3. MR.1280881 (95b:35036)

[56] Koji Mio, Tatsuki Ogino, Kazuo Minami, and Susumu Takeda, Modified nonlinear Schrödinger equation for Alfvén waves propagating along the magnetic field in cold plasmas, J. Phys. Soc. Japan 41 (1976), no. 1, 265-271. MR0462141 (57 \#2116)

[57] Koji Mio, Tatsuki Ogino, Kazuo Minami, and Susumu Takeda, Modified nonlinear Schrödinger equation for Alfvén waves propagating along the magnetic field in cold plasmas, J. Phys. Soc. Japan 41 (1976), no. 1, 265-271. MR0462141 (57 \#2116)

[58] Micheline Musette and Robert Conte, Analytic solitary waves of nonintegrable equations, Phys. D 181 (2003), no. 1-2, 70-79, DOI 10.1016/S0167-2789(03)00069-1. MR.2003796 (2004f:35148)

[59] A. F. Nikiforov, S. K. Suslov, and V. B. Uvarov, Classical orthogonal polynomials of a discrete variable, Springer Series in Computational Physics, Springer-Verlag, Berlin, 1991. Translated from the Russian. MR1149380 (92m:33019)

[60] S. Novikov, S. V. Manakov, L. P. Pitaevskiı̌, and V. E. Zakharov, Theory of solitons, The inverse scattering method; Contemporary Soviet Mathematics, Consultants Bureau [Plenum], New York, 1984. Translated from the Russian. MR779467 (86k:35142)

[61] B. Øksendal, Stochastic differential equations, Springer-Verlag, Berlin, 2000.

[62] S. M. Rytov, Yu. A. Kravtsov, and V. I. Tatarskiu, Principles of statistical radiophysics. 3, Elements of random fields; Springer-Verlag, Berlin, 1989. Translated from the second Russian edition by Alexander P. Repyev [A. P. Rep'ev]. MR 1002949 (90h:85005)

[63] W. van Saarloos, Front propagation into unstable states, Phys. Rep. 386 (2003), 29-222.

[64] Wim van Saarloos and P. C. Hohenberg, Fronts, pulses, sources and sinks in generalized complex Ginzburg-Landau equations, Phys. D 56 (1992), no. 4, 303-367, DOI 10.1016/01672789(92)90175-M. MR1169610 (93h:35195)

[65] B. Sanborn, S. K. Suslov, and L. Vinet, Dynamic invariants and Berry's phase for generalized driven harmonic oscillators, J. Russ. Laser Res. 32 (2011), no. 5, 486-494.

[66] G. A. Siviloglou and D. N. Christodoulides, Accelerating finite energy Airy beams, Opt. Lett. 32 (2007), no. 2, 979-981.

[67] G. A. Siviloglou, J. Broky, A. Dogariu, and D. N. Christodoulides, Observation of accelerating Airy beams, Phys. Rev. Lett. 99 (2007), 213901 (4 pages).

[68] Ronald Smith, Giant waves, J. Fluid Mech. 77 (1976), no. 3, 417-431. MR.0436757 (55 \#9696)

[69] E. Suazo and S. K. Suslov, Soliton-like solutions for nonlinear Schrödinger equation with variable quadratic Hamiltonians, J. Russ. Laser Res. 33 (2012), no. 1, 63-82.

[70] Sergei K. Suslov, On integrability of nonautonomous nonlinear Schrödinger equations, Proc. Amer. Math. Soc. 140 (2012), no. 9, 3067-3082, DOI 10.1090/S0002-9939-2011-11176-6. MR2917080

[71] Masayoshi Tajiri, Similarity reductions of the one- and two-dimensional nonlinear Schrödinger equations, J. Phys. Soc. Japan 52 (1983), no. 6, 1908-1917, DOI 10.1143/ JPSJ.52.1908. MR.710726 (84h:35154) 
[72] W. Tang and A. Mahalov, Stochastic Lagrangian dynamics for charged flows in the E-F regions of ionosphere, Physics of Plasmas 203 (2013), 032305 (11 pages).

[73] V. I. Talanov, Focusing of light in cubic media, JETP Lett. 11 (1970), 199-201.

[74] Terence Tao, A pseudoconformal compactification of the nonlinear Schrödinger equation and applications, New York J. Math. 15 (2009), 265-282. MR2530148 (2010h:35379)

[75] Sergey Yu. Vernov, Construction of special solutions for nonintegrable systems, J. Nonlinear Math. Phys. 13 (2006), no. 1, 50-63, DOI 10.2991/jnmp.2006.13.1.5. MR2217118 (2006m:34222)

[76] S. Yu. Vernov, Elliptic solutions of the quintic complex one-dimensional Ginzburg-Landau equation, J. Phys. A 40 (2007), no. 32, 9833-9844, DOI 10.1088/1751-8113/40/32/009. MR2370547(2008k:35456)

[77] M. B. Vinogradova, O. V. Rudenko, and A. P. Sukhorukov, Theory of waves, Nauka, Moscow, 1979 [in Russian]. MR1127872 (92j:00028)

[78] S. N. Vlasov and V. I. Talanov, The parabolic equation in wave propagation theory (on the 50th anniversary of the first publication) (Russian, with English and Russian summaries), Izv. Vyssh. Uchebn. Zaved. Radiofiz. 38 (1995), no. 1-2, 3-19, DOI 10.1007/BF01051853; English transl., Radiophys. and Quantum Electronics 38 (1995), no. 1-2, 1-12 (1996). MR1427164 (97j:78019)

[79] Miki Wadati, Heiji Sanuki, Kimiaki Konno, and Yoshi-Hiko Ichikawa, Circular polarized nonlinear Alfvén waves - a new type of nonlinear evolution equation in plasma physics, Conference on the Theory and Application of Solitons (Tucson, Ariz., 1976). Rocky Mountain J. Math. 8 (1978), no. 1-2, 323-331. MR0496220 (58 \#14796)

[80] Miki Wadati and Kiyoshi Sogo, Gauge transformations in soliton theory, J. Phys. Soc. Japan 52 (1983), no. 2, 394-398, DOI 10.1143/JPSJ.52.394. MR700302 (84g:35159)

[81] V. E. Zakharov and A. B. Shabat, Exact theory of two-dimensional self-focusing and onedimensional self-modulation of waves in nonlinear media (Russian, with English summary), Ž. Èksper. Teoret. Fiz. 61 (1971), no. 1, 118-134; English transl., Soviet Physics JETP 34 (1972), no. 1, 62-69. MR0406174(53 \#9966)

School of Mathematical and Statistical Sciences, Arizona State University, Tempe, ARIzONA 85287-1804

E-mail address: mahalov@asu.edu

School of Mathematical and Statistical Sciences, Arizona State University, Tempe, ARIZONA 85287-1804

E-mail address: sks@asu.edu

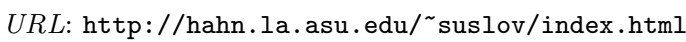

